\title{
Circulaire déterminant les conditions techniques auxquelles doivent satisfaire les distributions d'énergie électrique
}

Cette circulaire, en date du 30 avril 1927, a été publiée au Journal officiel du 23 juillet 1927 , pages 7634 à 7642 . L'arrêté auquel elle se réfère est publié à la suite, pages 7642 à 7652 (1).

Nous reproduisons ci-dessous le texte de la circulaire; celui de l'arrèté sera donné dans le prochain numéro.

le ministre des Travaux publics à M. le préfet du département de....

Paris, le 30 avril 1927.

J'ai l'honneur de vous adresser ci-joint ampliation d'un arrêté en date du 30 avril 1927 par lequel j'ai déterminé, d'accord avec mon collègue $M$. le ministre du Commerce et de l'Industrie, conformément à l'article 19 de la loi du 15 juin 1906 et après avis du Comité d'Electricité, les conditions techniques auxquelles doivent satisfaire les distributions d'énergie électrique au point de vue de la ićcurité des personnes et des services publics intéressés.

Je vous adresse, en même temps, les instructions nécessaires pour vous permettre d'en assurer l'application.

Le nouvel arrêté et la présente circulaire abrogent et remplacent l'arrêté et la circulaire du 30 avril 1924 (2).

L’arrêté débute par un préambule destiné à définir les installations auxquelles s'applique le présent arrêté. Ces installations sont celles qui constituent les ouvrages proprement dits de distribution d'énergie électrique et celles qui tonchent à la traction électrique.

Pour éviter toute confusion, on a systématiquement réservé le terme " alimentation " aux installations de la distribution d'énergie électrique spéciales à l'alimentation de la traction, le terme "contact "aux installations spéciales de prise de courant de traction et le terme " distribution " aux installations proprement dites de distribution d'énergie électrique.

Ainsi, des canalisations aériennes, souterraines ou, d'une façon plus générale, des ouvrages faisant partie d'installations de distribution d'énergie électrique dépendant d'un réseau de traction seront dénommés "canalisations aériennes d'alimentation " "canalisations souterraines d'alimentation ", "ouvrages d'alimentation ". Des canalisations aériennes, souterraines ou des ouvrages dépenlant d'installations proprement dites de distribution d'énergie électrique seront dénommés "canalisations aériennes de distribution ", "canalisations souterraines de distribution ", " ouvrages de distribution $n$. Lorsqu'il $\mathrm{y}$ a transformation du courant, la séparation entre le réseau de distribution et le réseau d'alimentation a lieu aux sous-stations et postes de transformation.

L'arrêté s'applique à tous les ouvrages empruntant en un point quelconque de leur parcours, le domaine public, ainsi qu'aux ouvrages établis exclusivement sur des terrains privés et s'approchant à moins de 10 mètres de distance horizontale d'une ligne de télécommunication préexistante; mais il ne s'applique ni aux usines de production d'énergie. à moins qu'elles ne fassent partie intégrante d'une concession, ni aux ouvrages d'utilisation situés dans les usines ou autres immeubles. Ces usines ou ouvrages d'utilisation sont soumis aux dispositions du décret du 1 er octobre 1913 , édicté en exécution des lois des 26 novembre 1912 et 31 décem-

(1) Cette circulaire et cet arrêté abrogent, ainsi qu'il y est dit, la circulaire el l'arreté du 30 avril 1924, qui eux-mêmes abrogeaient la circulaire et l'arrêté du 30 juillet 1921 . Rappelons que les textes de la circulaire et de l'arrêté de 1924 ont été reproduits dans la Revue générale de l'Electricité, 19 et 26 juillet 1924 , t. xvi, p. 125-136 et 171-183 et ccux de la circulaire et de l'arrêlé de 1921 dans la Revue générale de l'Electricité, 8,15 et 22 octobre 1921 , t. $x$, p. $496-504,538-543$ et $578-584$.

(2) Il est rappelé que les arrètés et circulaires antérieurs abrogeaient et remplaçaient, à l'exception de la circulaire du $1^{\text {er }}$ septembre 1909 sur les élagages, toutes les instructions techniques antérieurement en vigueur, notamment l'arreté préfectoral du 15 septembre 1893 , les instructions techniques annuelles émanant de l'administration des Postes et des Télégraphes et les dispositions techniques de l'instruction du jer février 1907, relative à la traversée des chemins de fer. bre 1912 sur l'hygiène et la sécurité des travailleurs dans les établissements industriels.

L'arrêté ne contient aucune prescription relative à la protection des sites que mentionne l'article 19 de la loi du 15 juin 1906.Je ne doute pas que les ingénieurs auront le plus grand souci de veiller à ce que l'établissement des ouvrages ne compromette pas le caractère artistique ou pittoresque des monuments, des paysages ou des rues de ville; il peut néanmoins être utile, toutes les fois que la situation le comportera, de consulter les fonctionnaires ou les commissions chargés, dans chaque circonscription administrative, de veiller à la conservation des monuments et des sites.

A cet égard, il sera bon que les ingénieurs se mettent en rapport avec l'architecte départemental lorsque les projets seront de nature à modifier l'aspect des rues ou des promenades des villes. Si les travaux projetés intéressent un immeuble classé parmi les monuments historiques en vertu de la loi du 30 mars 1887, il pourra être utilement fait appel à l'architecte ordinaire des monuments historiques; s'ils intéressent un paysage pittoresque, il $\mathrm{y}$ aura lieu, pour vous, de saisir la commission instituée dans votre département par la loi du 21 avril 1906, sur la conservation des sites et des monuments naturels.

Après le préambule, l'arrêté est divisé en trois titres dont le premier comprend les dispositions applicables aux ouvrages de distribution (et d'alimentation, ainsi qu'il est dit au préambule), le second, les dispositions applicables aux installations de traction électrique et le troisième, les dispositions diverses, notamment conditions et délais d'application de certaines dispositions de l'arrèté.

Comme on le verra, la présentation de l'arrêté a subi d'importantes modifications dans le but d'en rendre la lecture plus aisée. Des modifications de fond ont été également introduites, principalement dans le but de renforcer les prescriptions relatives à la sécurité. Celles de ces moidfications dont le sens est évident ne feront l'objet d'aucun commentaire dans la présente circulaire.

Titre Ier. - Dispositions applicables alix ouvrages DE DISTRIBLTION.

Le titre Ier concernant les dispositions applicables aux ouvrages de distribution (et d'alimentation ainsi qu'il est dit au préambule) comporte cinq chapitres, savoir :

Chapitre I I $^{\text {er. }}$ - Classement des ouvrages et prescriptions générales relatives à la sécurité.

Chapitre II. - Dispositions communes aux trois catégories (l'article classe, en effet, désormais les ouvrages en trois catégories au lieu de deux).

Chapitre III. - Dispositions spéciales aux ouvrages de première catégorie.

Chapitre IV. - Dispositions spéciales aux ouvrages de deuxième catégorie.

Chapitre $Y$. - Dispositions spéciales aux ourrages de troisième catégorie.

Cette nouvelle répartition des matières entre les chapitres, qui sont eux-mêmes divisés en sections, facilitera la consultation de l'arrêté.

Ch. Ier. - Classement DES OUVRages ET PRESCRIPTIONS GÉNÉRALES RELATIVES A LA SÉCURITÉ.

Le chapitre Ier contient les dispositions techniques générales applicables à tous les systèmes de distribution : il donne lieu aux observations suivantes :

Art. ler. - Les installations sont classées en trois catégories suivant la plus grande tension de régime existant entre les conducteurs et la terre, ou, dans les distributions triphasées, entre les conducteurs et le point neutre, que ce point neutre soit, ou non, effectivement mis au sol. Il est rappelé à ca sujet què, si le point neutre 
dune distribution triphasé, dont la tension entre phases est $U_{\mathrm{er}}$, n'est pas matérialisé, la teusion par rapport au point neutre, qui sert de base pour définir la catégorie de la distribution, a pour villeur $\frac{l_{\mathrm{eff}}}{1 \frac{1}{3}}$

La répartition de la première catégorie en deux subdivisions $B_{1}$ et $B_{2}$. en cas de courant alternatif, a été maintenue en raison du fait que les installations de la subdivision $B_{2}$ dans les immeubles, doivent ètre exécutées avec plus de soins encore que celles de la subdivision $B_{1}$ : bien que ces installations ne soient pas directement réglementées par le présent arrìté. les services de contròle peuvent avoir à en comnaitre, en vertu des dispositions de l'article 19 des cahiers des charges de concessions conformes au type.

Il importe d'ailleurs de ne pas perdre de vue que les ouvrages de deuxième et de troisième catégories ne sont pas les seuls qui puissent présenter des dangers; les limites indiquées pour la tension maximum de première catégorie correspondent aux installations usuelles, où les accidents se produisent le plus rarement, mais il a été constaté que, dans certaines circonstances, des courants dont la tension est très inférieure à la limite adoptée. ont occasionné des électrocutions. Vous aurez à tenir compte de ce fait dans l'étude des installations de première catégorie.

La troisième catégorie comprend tous les ouvrages dont les tensions rentrent dans celles de l'ancienne division $H$ de deuxième catégoric. Les dispositions de l'arrêté prëmnent ainsi une plus grande netteté.

\section{Ch. II. - Dispositions conmunes anx trois caígorifs.}

\section{Section I. - Supports, canalisations aériennes.}

Art. 3. - Les conditions exigées à l'article 6 pour la résistance mécanique des ouvrages donneraient, en ce qui concerne les supports en bois, une sécurité illusoire si leur implantation dans le sol n'était pas faite avec précaution, notamment à une profondeur suffisante corrélative de leur hauteur.

Art. 4. - Les essais des isolateurs ne peuvent être pratiquement faits sur une ligne établie ; conformément à la pratique courante de l'industrie, les isolateurs seront essayés à l'usine avant livraison; la service de contrôle pourra exiger la production du procès-verbal des essais.

Les essais d'isolateurs nécessitant des prescriptions assez longues et d'ailleurs modifiables suivant les progrès de la construction, il y aura avantage pour les services de contrôle à se guider sur les règles d'essais établies par les syndicats professionnels intéressés lorsqu'ils auront à apprécier les procès-verbaux d'essais en usine qui leur seront communiqués.

Art. 5. - Il peut être utile de rappeler, pour éviter les divergences éventuelles d'interprétation, que la section d'un câble est la somme des sactions droites des brins qui la composent.

L'arrêté du 30 avril $192 t$ avait déjà réduit à $15^{\circ}$, pour les lignes, l'angle minimum pour la traversée des routes $(\$ 3)$, afin de réduire l'angle de la brisure dans la direction générale de la ligne. Cette brisure constitue, en effet, malgré la consolidation des supports, un point faible dans les canalisations et la diminution de la sécurité qui en résulte l'emporte, tant que l'angle de la traversée ne devient pas très faible, sur l'avantage qu'il peut y avoir à réduire la longueur de conducte'irs dominant la voie publique. En outre, l'emploi d'alignements droits permet, en général, de réduire le nombre des appıis. Aussi, le présent arrêté maintient-il ces dispositions.

Les épissures et soudures interdites ( $\$ 4$ ) dans la traversée des voies publiques désignées au paragraphe 3 et dans les portées contiguës pauvent être autorisées à titre provisoire comme moyen de réparation. Cette interliction ne s'oppose pas à ce qu'il soit fait usage, dans les portées contiguës à la traversée, de manchons de jonction présentant une résistance mécanique au moins égale à calle du conducteur.

Dans les lignes à supports en bois, la disposition qui consiste à compenser la traction des conducteurs sur un poteau d'angle ou sur le dernier poteau de la portée extrême par un hauban, constitué par un fil attaché à la partie supérieure du poteau et ancré dans le sol au moyen d'un piquet implanté suivant la bissectrice de l'angle ou dans le prolongement de la portée terminus, est dangereuse lorsque le lauban vienl à être rendu libre fortuitement par le desancrage du piquet en raison des déplacements qu'il subil alors sous laction du venl et par le fail des passants. Elle l'esl surtout lorsque le point d'attache du hauban est au-dessus des conductcurs, les risques de contacl du hauban avec un conducteur étant très grands dans ce cas et son électrisalion devant prescrue fatalenent se produire. Aussi, bien que l'arrêté n'interdise pas lo haubannage, qui peut ctre motivé dans certains cas exceptionnels, vous voudrez bien en réduire l'emploi le plus possible, et, cuand vous l'autoriserez, veille à te que le point d'attache du haubannage soit toujours au-dessous des conducteurs.

Art. 6. ... I. article b définit les conditions dans lesfuclles doivent itre calculées les dimensions des conducteurs, supports ef ferrures des ouvrages de distribution.

Il y a lieu de tenir compte dans ce calcul, non seulemenl des charges permamentes que les organes ont à supporter, mais aussi des charges accidentelles qui peuvent se produire sous l'action du vent. Ces charges accidentelles peuvent d'ailleurs varier suivant la température. Par les temps froids, la fleche des conducteurs diminue, ce qui est défavorable ì la sécurité, mais par contre, en général, dans ces circonstances, la violence du vent n'atteint pas le maximum constaté avec des températures moyennes. 11 conviendra de faire le calcul dans les deux hypotheses et de retenir le résultat trouvé dans le cas le plus défavorable.

Pour les prlònes en treillis, la pression du vent sera prise entière sur la première face frappée el réduite pour la face arrière. Ia formule de réduction à employer sera celle des règlements ministériels en vigueur pour le calcul des ponts métalliques.

Il n'y a pas lieu, dans la plupart des cas, d'envisager l'hypothese d'une couche de verglas recouvrant les conducteurs, cette couche se produisant plus rarement sur les conducteurs d'énergie que sur les lignes de télécommunication en raison de la chaleur développéc par le passage du mène courant. Toutefois, il peut, dans certaines régions, par exemple en paýs de montagne, se produire des dépòts de verglas sur les conducteurs : dans ces cas spéciaux, il y aura lieu d'en tenir compte dans les calculs justificatifs.

En outre, il a paru nécessaire de préciser dans un deuxième paragraphe que, dans le cas où les supports sont munis de maconneries de fondations, ces fondations sont établies suivant les règles de l'art.

Cette addition a pour but d'éviter qu'on puisse interpréter les dispositions des paragraphes précédents comme nécessitant l'emploi de maçonneries de fondations pour les supports, alors qu'elles peuvent ètre inutiles, et d'autre part, qu'on exige pour ces maçonneries, lorsqu'elles existent, des coefficients de sécurité rigidement déterminés, qui n'apparaissent pas comme indispensables ou des matériaux de qualité exceptionnelle.

\section{Section II. - Canalisations sonterraines.}

Art. 7. - L'armature métallique d'un câble souterrain peut suffire comme protection mécanique de celui-ci; mais les câbles souterrains ne sont pas nécessairement des câbles armés; le paragraphe 2 prévoit, dans ce cas, la nécessité d'une protection mécanique.

Les câbles souterrains doivent ètre des meilleurs modèles connus, comportant une chemise de plomb sans soudure. Il est à peine besoin de signaler que cette disposition a pour but de préciser le système suivant lequel est établic la chemise de plomb, mais non de spécifier qu'il ne devra y avoir trace d'ancune soudure sur cette chemise ; en particulier. les reprises de presse ne peuvent être toujours évitées clans la fabrication et ne sauraient être interdites.

Section V. - Jisposilions speciales applicables ì la trabersie des cours d'eau al des canam de navigation.

La section $V$ détermine les conditions spéciales auxquelles doivent satisfaire les ouvrages à la traversce des cours d'eau et des canaux de navigation.

Aucune disposition spéciale n'a été prévue pour le cas où les conducteurs traversent la voie d'cau aut-dessus d'un passage supérieur; il est bien évident que les conditions spéciales exigées par l'existence de la voie d'eau ne s'appliquent pas en pareil cas.

Il va de soi que sur certains cours d'eau frécquentés par des batiments de mer, le service du controle aura à imposer, pour 
les traversées, les conditions spéciales nécessaires dans l'intérèt de la navigation el de la sécurité.

\section{Section VI. - Dispositions spéciales applicables à la traversée el au voisinage des lignes d'énergie éleclrique ou des conduiles d'eau, de gaz on d'air compriné.}

Art. 22, \$1 $1^{\mathrm{er}}$. - L'installation sur les mêmes poteaux ou pylônes de ligno. de $1^{\text {re }}$ catégorie et de lignes de $2^{\text {e }}$ et de $3^{\mathrm{e}}$ catégories n'est pas interdite; mais ce dispositif no pourra être admis que si l'impossibilité d'employer des supports différents est reconnue par le scrvice du contrôle, et jamais sans qu'interviennent d'autres raisons que ]'économie.

Il y a lieu de remarquer, en ce qui concerne l'application de la disposilion générale du paragraphe 2, qu'il est nécessaire que la distance à laquelle peut arriver l'appareil de prise de courant ne soit pas inutilement augmentée en cours d'exploitation par le concestionnare de la voie ferrée.

\section{Section VII. - Dispositions spéciales applicables à la} traversét des lignes de chemins de fer.

Il importe de ne pas perdre de vue que les dispositions prescrites ne concernent pas les traversées de voies ferrées énoncées dans le renvoi en note au titre de la sertion VII, qui ne constituent pas les traversées de chemin de fer visces par la présente section.

En outre, il est rappelé qu'une canalisation souterraine, empruntant la voie publique pour traverser un chemin de fer sous un passage inférieur sans intéresser les ouvrages du chemin de fer, peut être établic sans intervention du service du contrôle du chemin de fer et sans arrèté spécial d'autorisation pour la traversée.

H Toutefois, avant d'entreprendre les travaux de terrassement aux abords de l'ouvrage, il conviendra d'en donner avis aux représentants de l'administration exploitant le chemin de fer

Division I. - Traversée des lignes de chemin de fer des grands réseaux d’intérêt général.

Art. 25. - Les passages à niveau ne sont pas classés parmi les points qui doivent être choisis de préférence pour la traversée des chemins de fer; la traversée aux passages à niveau crée, en effet, un risque pour la circulation publique. Il peut être avantageux, toutefois, au lieu d'établir une traversée en pleine voie, de la placer à proximité d'un passage à niveau pour qu'elle puisse être surveillée par le garde-barrière. Mais ce n'est pas là une obligation ; il appartient aux services de contrôle d'adoptèr la solution la plus conforme aux intérèts en présence.

Art. 26 et $2 \%$. - Il n'a pas paru nécessaire de fixer une limite pour la densité maximum du courant dans les canalisations aériennes et souterraines. Les nécessités industrielles obligent, en effet, les intéressés à adopter cles densités de courant bien inférieures à celles qui pourraient compromettre la sécurité.

Art. 26, \$ 4. - Il y a lieu de noter que des isolateurs ne sont pas compris dans les organes de supports visés au présent paragraphe.

Les maçonneries de fondations qui, d'après l'article 6, paragraphe 2, doivent être établies conformément aux règles de l'art, se trouvent définies par la nćcessité de satisfaire aux coefficients de stabilité fixés par l'arrêté.

Enfin, il est spécifié que les coefficients de stabilité ne seront pas exigés pour les appuis scellés dans le rocher.

Art. 26, \$5. - Ce paragraphe vise les distributions qui traversent ou empruntent les lignes de chemins de fer d’intérêt général el prescrit des vérifications périodiques de ces installations.

Ces vérifications devront faire l'objet de procès-verbaux mais il ne paraìt pas utile d'établir à ect effet un modèle spécial et uniforme. Ies constatations faites varieront évidemment, suivant le cas, de telle sorte gu'il vaut mieux laisser au service de contròle le soin de rédiger le procès-verbat à son gré.

Drvision II. -- Traversée des lignes de chemins de fer des réseaux secondaires d'intérèt général et des réseaux d'intérèt local.

Art. 28. - - Les dispositions spéciales aux traversées par les canalisations des diverses catégories seront reprises dans les chapitres suivants.
Section VIII. -.. Prolection des lignes de tétécommunication.

Le paragraphe 3 de l'article 29 précise certaines précautions supplémentaires dans le cas où une ligne de distribution croise simultanément, dans la même portée, une ligne de contact et des fils de télécommunication. Ces précautions supplémentaires ne devront d'ailleurs être employées que s'il n'est pas possible de supprimer ces doubles croisements, par exemple en plaçant un appui intermédiaire.

Quand les lignes de télécommunication ne peuvent être placées au-dessous des conducteurs d'énergie, il convient de les consolider, s'il y a lieu, pour éviter leur rupture, indépendamment du dispositif de garde solidement établi entre les deux sortes de conducteurs. Au sujet de ce dispositif de gardr, je vous rappelle qu'il convient de renoncer d'une facon définitive aux baguettes de protection en bois placées sur le fil de contact; ce système de protection ne devra donc pas etre admis pour les installations nouvelles; pour les anciennes, où il est encore en usage, son emploi devra être abandomé progressivement, at fur et à mesure de la mise hors service des dispositifs existants.

\section{Section IX. - Entretien des ouvrages.}

Art. 31. - Il est rappelé que les conditions d'application de l'article 31 , relatif à l'élagage des plantations ont été précisées par la circulaire du $1^{\text {er }}$ septembre 1909 , à laquelle il y a lieu de se référer. Il importe de faciliter le plus fossible l'exécution des élagages en raison des nombreuses interruptions de service dont la cause est uniquement attribuable à des branches d'arbres.

\section{CH. III - Dispositio ss Spéciazes aux otvrages DE PREMIỲRE CATÉGORIE.}

Art. 33. - La mise au sol du point neutre et du conducteur neutre, s'il $y$ en a un, dans les distributions triphasées de première catégorie en étoile derient obligatoire dans la subdivision $B_{1}$, en vue d'améliorer la sécurité des installations de cette subdivision au cas où il se produirait un mélange avec une installation de catégorie supérieure, soit dans une canalisation, soit dans un transformateur ou tout autre appareil.

Ces mises au sol devront être, de préférence. effectuées en plusieurs points pour ascurer en permanence une bonne communication.

Art. 34. - L'arrêté précise la nature des voies publiques pour lesquelles il $\mathrm{v}$ a lieu de rapprocher les supports autant que possible, les services de contrôle conservant d'ailleurs une certaine latitude pour l'application de cette formule en s'inspirant des conditions locales. Les dispositions prévues pour les routes nationales ou départementales, les chemins de grande communication ou d'intérêt commun, sont applicables aux rues des villes ou villages qui bénéficient d'un de ces classements; il $\mathrm{y}$ aura lieu également de les appliquer aux voies urbaines dont la fréquentation sera particulièrement intense. Au surplus, il suffira, dans un grand nombre de cas, que l'un des appuis de la traversée soit au roisinage immédiat de la voie publique.

Art. 35. - Le point le plus bas des conducteurs est maintenu à $6 \mathrm{~m}$. le long et à la traversée des roies publiques pour les distributions de première catégorie. mais à la condition que le minimum prescrit soit observé strictement, mème pendant les plus grandes chaleurs de l'été (\$ $1^{\mathrm{er}}$.)

Il n'est fait d'exception que dans deux cas :

10 A la traverséc des ouvrages construits au-dessus des voies publicques, où une hauteur inférieure à $6 \mathrm{~m}$. peut ètre admise, pourvu que la sécurité soit assurée par un dispositif spécial de protection, mais sans que la hanteur libre de $4 \mathrm{~m}$. 30 ì réserver au-dessus de la chaussée puisse itre diminuée;

20 Le long et à la traverséc des chemins ou parties de chemins qui ne peuvent en aucun cas itre accessibles aux véhicules et sur lesquels, en raison de cette circonstance. les canalisations sont simplement tenues d'ètre, en vertu du paragraphe $1^{\text {er }}$ de larticle 5, hors de la portée du public.

Dans les parties en courbe des voies publiques, les poteaux ou pylônes devront être plus rapprochés que dans les alignements droits pour diminuer l'empietement en projection horizontale 
des conducteurs sur la voie publique; il importe déviter des contaets possibles avee des chargements élevés.

\section{Section III. - Dispositions spéciales applicables à la traverséc des lignes de chemins de fer.}

Drvisiox I. - - Traversée des lignes de chemins de fer des grands réseaux d’intérèt général.

Art. 38. - Larticle 38 précise que les appareils de coupuro ne doivent pas nécessairement ètre établis dans le voisinage immédiat de la traversée : il suffit que l'installation soit faite de manière qu'il soit possible de couper facilement le courant dans la traversée. en évitant tout retour de courant.

Art. $39, \$ 1^{\mathrm{er}}$ - - Le texte vise les cas particuliers, notamment celui des abords des gares, ou il peut y avoir un grand nombre de voies à traverser. Dans ce cas, il y a intérêt, au point de vue de la sécurité, à avoir un ou mème, sül $r$ a lieu, plusieurs supports intermédiaires.

Art. 39, \$ 3. - Le Comité d'Electricité estime qu'il n'y a pas lieu d'imposer un dispositif de protection d'une manière exclusive. Il a'reconnu qu'il existe un grand nombre de dispositifs satisfaisants permettant de doubler les conducteurs soit dans toute la portée de la traversée, soit an droit des isolateurs seulement. Il a donc estimé qu'il $y$ avait lieu d'en signaler quelques-uns à titre d'exemple sans en imposer alleun.

Une planche schématique (fig. 2) avec légende dome les explications nécessaires sur six dispositifs qui ont paru présenter une bonne garantie au point de vue de la sécurité.

\section{Section IV. - Protection des lignes de lélécommuntation.}

Art. $42, \$ 2$. - Les points d'appui des conducteurs de distributions doivent ètre assez rapprochés l'un de l'autre, de part et d'autre du croisement, pour qu'il n'y ait pas de flottement important de ces conducteurs.

Les branchements constitués au moven de conducteurs isolés sont assujettis aux mêmes conditions que les branchements en conducteurs nus aux traversées et clans le volsinage des lignes de télécommunication.

\section{Ch. IV. - Dispositions SpÉciales auX ouvrages DE DEUXIÈME CATÉGORIF.}

La mise au sol du point neutre des canalisations de deuxième catégorie n'est pas rendue obligatoire par l'arrêté.

\section{Section I. - Supports. Canalisations aériennes.}

Art. 45. - Dans les distributions de deuxième catégorie, les pylônes et poteaux métalliques et en béton armé devront être mis en communication avec le sol. Pour que cette protection soit suffisante en cas de chute de conducteur, vous aurez à exercer une surveillance minutieuse sur les conditions de cette mise au sol des supports. Je vous signale parmi les dispositifs susceptibles d'être parfois appliqués avantageusement, celui qui consiste à relier les pylônes par un fil supérieur et à ne mettre en communication directe avec le sol que ceux d'entre eux pour lesquels il est possible de trouver une bonne terre.

En ce qui concerne les pylônes et poteaux en béton armé, ce sont les supports d'isolateurs seuls que l'arrêté prescrit de relier au sol.

En ce qui concerne l'application du paragraphe 2, les deux dispositifs suivants peuvent être notamment employés pour empêcher, dans la mesure du possible, le public d'atteindre les conducteurs :

$1^{\circ}$ Fil de fer barbelé enveloppant le support dans le cas de poteaux en bois ou en ciment armé et les montants en fer dans le cas de pylônes métalliques, le fil de fer étant placé à partir de $2 \mathrm{~m}$. du sol, sur une hauteur d'un mètre, sur lacquelle il y aura dix tours de ronces en fil d'au moins deux millimètres de diamètre avec picots espacés de 60 millimètres;

$2^{\circ}$ Herses à piquants rigides entourant les supports. L'emploi les herses en tôle découpée dont on a rabattu certaines parties ne sera autorisé que si la tôle est assez rigide pour qu'on puisse ne pas rabattre les piquants à la main.

le dispositif empèchant l'ascension par l’intérieur pourra être, par exemple, un croisillon horizontal ou oblique.

Il convient de remarquer qu’aucun dispositif ne réussira à empècher d'une maniere absolue quelqu'un qui veut monter au poteau, d'y parvenir. Ce n'est que par l'éducation du public, qui peut ètre obtenue surtout dans les écolés, qu'on pourra diminuer ces tentalives.

Les supports des installations de deuxième calégorie devront porter l'inscription : "Danger de mort ", après les mots : "Défense absolue de toucher aux fils, mème tombés a terre ", afin qu'il apparaisse clairement aux yeux de tous que, si le danger de mort est réel et doit ètre explicitement signalé, il résulte du contact avec les fils ; cette inscription figurera sur une plaque dont je rléterminerai les caractéristiques générales.

Il est bien entendu que le remplacement des plaques actuellement posées par les nouvelles sera effectuć dans les conditions fixées par l'article 123.

Il est désirable, autant que possible, que les ligne's de deuxième catégorie, d'une part, et les lignes de télécommunication parallèles, d'autre part, ne soient pas placćes du mème côté des voies publiques.

La remarque suivante est rappelée en ce qui concerne le paragraphe 5 .

L'arrèté précise la nature des voies publiques pour lesquelles il $\mathrm{y}$ a lieu de rapprocher les supports autant que possible, les services de contrôle conservant d'ailleurs une certaine latitude pour l'application de cette formule en s'inspirant des conditions locales. Les dispositions prévues pour les routes nationales ou départementales, les chemins de grande communication ou d'intérêt commun sont applicables aux rues qui bénéficient d'un de ces classements; il y aura lieu également de les appliquer aux voies urbaines dont la fréquentation sera particulièrement intense. Au surplus, il suffira, dans un grand nombre de cas, que l'un des appuis de la traversée soit an voisinage immédiat de la roie publique.

Art. 46. - Le point le plus bas des conducteurs est maintenu a $6 \mathrm{~m}$. le long des voies publiques et à $8 \mathrm{~m}$. dans les traversées pour les distributions de deuxième catégorie, mais à la condition que le minimum prescrit soit observé strictement, même pendant les plus grandes chaleurs de l'été ( $\$ 1$ er).

Il n'est fait d'exception que dans deux cas :

10 A la traversée des ouvrages construits au-dessus des voies publiques, où une hauteur inférieure à $6 \mathrm{~m}$. peut être admise, pourvu que la sécurité soit assurée par un dispositif spécial de protection, mais sans que la hauteur libre de $4 \mathrm{~m} .30$ à réserver au-dessus de la chaussée puisse être diminuée:

$2^{\circ}$ Le long et à la traversée des chemins ou parties de chemins, qui ne peuvent, en aucun cas, être accessibles aux véhicules et sur lesquels, en raison de cette circonstance, les canalisations sont simplement tenues d'ètre, en vertu du paragraphe $1^{\text {er }}$ de l'article 5 , hors de la portée du public.

Dans les parties en courbe des voies publiques, les poteaux ou pylônes devront être plus rapprochés que dans les alignements droits pour diminuer l'empiètement en projection horizontale des conducteurs sur la voie publique; il importe d'éviter des .ontacts possibles avec des chargements élevés.

On peut réaliser les prescriptions du paragraphe 2 par divers dispositifs, notamment en placant sur les supports en question des cadres métalliques entourant tous les conducteurs (tels que les cadres de mise au sol), ou en prolongeant les ferrures supportant les isolateurs par des cornes métalliques de forme et de longueur appropriées, qui maintiennent ces conducteurs au cas où ils viendraient à abandonner l'jsolateur.

Les croquis traduisant les dispositions à adopter pour les conducteurs au voisinage d'un toit en pente et d'un toit en terrasse sont conformes à ceux de la figure 1 (art. 35).

Les dispositions de l'article 47 ne distinguent pas des autres les ouvrages en béton armé, auxquelles sont applicables, sans restriction, les instructions du 20 octobre 1906 relatives à J'emploi du béton armé pouvant, dans celles de leurs clauses où elles sont plus rigoureuses, être, sans inconvénient, amendées pour des pylônes construits en usine. 
Art. 48. - Dans les distributions de deuxième catégorie, les accidents peuvent nécessiter la coupure du courant dans le plus bref délai possible. $A$ cet effet, l'artícle 48 prévoít que chaque agglomération importante doit être reliée par un moyen de communicatín directe à l'usine génératrice ou au poste le plus voisin muni d'appareil de coupure. Le distributeur peut, pour réaliser cette liaison, faire usage de télécommunication, avec ou sans fil, ou avoir recours à d'autres moyens, par exemple munir le personnel de surveillance de moyens de transport rapides (automobiles, bicyclettes, etc.). Il appartiendra au service du contrôle d'apprécier les propositions faites à cet effet par le distributeur.

Dans le cas où la distribution est munie d'appareils de coupure à l'entrée de chaque agglomération, l'installation pourra être considérée comme répondant à la prescription de l'article 48 , a la condition, toutefois, que le distributeur ait pris toutes les mesures nécessaires pour que ces appareils puissent être manœuvrés efficacement quand il en sera besoin.

Dans les installations de traction de deuxième catégorie, l'article 48 prévoit que les conducteurs aériens devront être protégés par des dispositifs destinés à limiter l'intensité du courant.

\section{Section II. -.. Sous-stations, postes de transformation et installations diverses.}

Art. $49, \S 1^{\mathrm{er}}$ - - Pour garantir la sécurité du personnel amené à travailler dans les locaux non gardés où sont installés des transformateurs de deuxième catégorie, en cas d'incendie ou d'explosion, il est nécessaire que les portes, lorsqu'elles sont à rabattement, ne s'ouvrent pas vers l'intérieur. Or, ces locaux sont fréquemment installés sur une voie publique ou en bordure d'une pareille voie et les règlements de voirie interdisent généralement l'ouverture des portes vers l'extérieur. Il est évident que les motifs qui ont déterminé cette disposition doivent céder devant la question primordiale de sécurité du personnel; il y aura donc lieu en pareil cas, d'autoriser l'ouverture des portes vers l'extérieur, en veillant toutefois à ce que la saillie qui résultera du rabattement de la porte sur le mur de façade soit réduite au minimum.

Art. 50. - L'arrêté prévoit la protection des canalisations nues par des grillages ou des écrans en métal ou en matière isolante, en vue d'accroître la sécurité du personnel obligé d'y travailler.

S'il est fait emploi de couches de peinture pour différencier les conducteurs, on pourra employer une couleur déterminée pour chaque phase, quelle que soit la position respective des conductèurs, sans que cette indication doive être considérée comme imposant ce procédé de préférence à tout autre.

Art. 51, § 2. - De la prescription ainsi conçue : "L'accès de ce passage est défendu par une porte fermant à clef ", on a cru parfois pouvoir conclure que ladite porte devalt rester fermée à clef en permanence. Une pareille interprétation serait erronée, car c'est avec intention qu'il est écrit "fermant à clef " et non "fermé à clef ". La porte doit pouvoir être fermée à clef lorsqu'aucune surveillance ne peut être exercée sur l'accès qu'elle défend. Mais il arrivera fréquemment que les nécessités de l'exploitation obligent à la laisser ouverte, et il n'en résultera aucun inconvénient si l'accès est, pendant ce temps, l'objet d'une surveillance pour que seul le personnel qui a mission de pénétrer dans la partie arrière du tableau puisse le faire.

\section{Section IV...-Disposilions spéciales applicables} à la traversie des chemins de fer.

Drvision I. - Traversée des lignes de chemins de fer des grands réseaux d'intérêt général.

Art. 55 et 56 . - Les remarques formulées précédemment au sujet des articles 38 et 39 sont applicables également aux articles 55 et 56 .

\section{Section V. - Protection des lignes de télécommunication.}

Art. 58. - La section V traite de la protection des lignes de télécommunication et appelle les observations suivantes :

Le voisinage de ces lignes et des lignes de distribution doit etre l'objet d'une attention particuliere, l'indlcation d'une distance minimum de $1 \mathrm{~m}$. ou de $2 \mathrm{~m}$. entre ces lignes, sauf lorsque les con- ducteurs d'énergie sont fixés sur toute leur longueur, n'exclut nullement l'adoption d'un plus grand écartement s'il est pratiquement et raisonnablement réalisable.

Il a été rappelé, au paragraphe $1^{\mathrm{e}}$, que les canalisations d'énergie ne doivent produire aucune perturbation nuisible par induction (électrique ou électromagnétique) sur les lignes de télécommunication.

L.e premier alinéa du paragraphe 2 de l'article 50 spécifie qu'aux points de croisement, les conducteurs de distribution sont, autant que possible, placés au-dessus des fils de télécommunication. Il doit être bien entendu que cette disposition supérieure des conducteurs d'énergie est à réaliser sauf impossibilité.

D'autre part, il convient de chercher à supprimer les croisements toutes les fois qu'il est possible de le faire moyennant une modification des lignes de télécommunication n'entraînant qu'une dépense raisonnable à la charge des distributeurs.

En ce qui concerne le dispositif de garde mentionné in fine du paragraphe 2 , il y a lieu de signaler que le poteau ou pylone s'il est placé entre la canalisation d'énergie et la ligne de télécommunication, constitue un dispositif de garde suffisant.

Au sujet de l'article 59, il est rappelé que si les lignes de télécommunication sont, dans le cas visé, assimilées aux lignes de deuxième catégorie, il ne saurait être question de leur imposer l'emploi d'isolateurs susceptibles de tenir la mème tension que ceux de la ligne d'énergie.

En outre, une exception aux prescriptions de l'article 59 a été apportée en ce qui concerne les parties de lignes de télécommunication montées sur des supports particuliers et suffisamment séparées électriquement des parties montées sur les mêmes appuis que la canalisation d'énergie.

\section{Section VI. - Exploitation des distributions.}

Art. 60 et 79. - Ces deux articles qui visent respectivement les distributions de $2^{\mathrm{e}}$ et de $3^{\mathrm{e}}$ catégorie, prescrivent pour la manœuvre des interrupteurs aériens diverses mesures de sécurité qui se justifient d'elles-mêmes et qu'il importe de ne pas omettre.

Quant aux précautions à prendre pour l'exécution des travaux sur les conducteurs ou à leur voisinage, elles sont fixées par l'arrêté ministériel du 19 mars 1927 , déterminant les mesures de prodection applicables dans les chantiers de construction et d'entretien tes entreprises de distribution d'énergie électrique, auquel on devra se reporter en ce qui les concerne. Je me bornerai ici à appeler l'attention sur un point qu'il importe de ne pas perdre de vue quand on doit exécuter un travail sur une ligne d'énergie.

Les ingénieurs à qui revient le soin d'établir des consignes pour l'exécution de ce travail doivent, en effet, se préoccuper des dangers des courants d'induction. Il peut arriver en effet que les fils de télécommunication ou les conducteurs d'énergie sur lesquels on travaille se prolongent au delà de la section sur laquelle le courant est coupé. S'ils restent alors, sur un certain parallélisme, au voisinage d'autres conducteurs industriels, les disjonctions ou ies mises en charge de ceux-ci sont susceptibles, par voie indirecte, de produire des forces électromotrices, donnant lieu à des ondes de tension dangereuse, atteignant la section où l'on se croyait en sécurité, pour y avoir supprimé l'alimentation.

Art. 61 et 80. - Ces deux articles fixent les règles à suivre pour l'affichage des prescriptions de sécurité dans les distributions de $2^{\mathrm{e}}$ et de $3^{\mathrm{e}}$ catégorie.

Quant aux soins à donner aux victimes des accidents dus à l'électricité, l'affichage doit en être fait suivant les prescriptions du décret du 23 janvier 1927 qui en a fixé les règles.

\section{Ch. V. Dispositions spéciales atix ouvrages DE $3^{\text {e }}$ CATÉgorie.}

Ces ouvrages sont soumis aux prescriptions du chapitre II du titre $1^{\mathrm{er}}$ et, en outre, aux dispositions du présent chapitre.

En dehors de toutes les conditions prescrites, l'attention des exploitants doit être spécialement attirée sur l'importance d'une construction très soignée des ouvrages, qui constitue en définitive l'une des principales garanties de sécurité.

La mise au sol du point neutre des canalisations de troisième catégorie n'est pas rendue obligatoire par l'arrèté. Cette mise au 
sol est assurément recommandable dans presque tous les cas, mais la technique n'est pas encore assez fixée pour yu'il soit admissible le l'imposer dès à présent.

Il n'a pas paru possible d'interdire absolument la traversée des agglomérations par des canalisations de troisième catégorie, mais il y aura lieu d'éviter ces traversées toutes les fois qu'il y aura possibilité de le faire et mème, clans la mesure du possible, la proximité des bàtiments.

Art. 63. - Les remarques formulées au sujet de l'article 45 s'appliquent au présent article; les points suivants sont en outre signalés :

Poteaux en bois. - Bien que les poteaux en hois ne soient pas exclus dans l'établissement des lignes de troisième catégorie, leur emploi devra ètre limité de préférence aux installations provisoires; pour les installations définitives, ils ne devront ètre admis que s'ils sont montés sur socle métallique ou en béton.

Mise au sol des supports. - La mise au sol des pylònes et ouvrages devra ètre réalisée par l'intermédiaire de conducteurs de section suffisante : $30 \mathrm{~mm}^{2}$ au moins sils sont en cuivre et $50 \mathrm{~mm} \mathrm{~m}^{2}$ au moins s'ils sont en fer.

Le point le plus bas des conducteurs est maintenu à $6 \mathrm{~m}$. au-dessus du sol des propriétés privées et le long des parties des voies publiques accessibles aux véhicules, et à $8 \mathrm{~m}$. dans les traversées de ces voies, mais à la condition que les minima prescrits soient observés strictement, même pendant les plus grandes chaleurs de l'été.

Encadrement de routes. - Il est indispensable de prévoir l'emplacement des supports de façon à respecter la distance prescrite entre les conducteurs et le sol.

Tracé. - Les angles des lignes constituant toujours des points faibles, les tracés les plus rectilignes devront autant que possible être recherchés.

Attache de sécurité. - Dans la traversée des routes nationales, départementales, des chemins de grande communication et d'intérèt commun, ainsi qu'aux croisements de circuits de télécommunication, comme aux traversées de voies ferrées, électrifiées ou non, et des lignes électriques préexistantes, il peut être utile de doubler les conducteurs au droit et de part et d'autre des isolateurs par une bretelle lorsqu'il s'agit d'une canalisation avec isolateurs suspendus.

Cette attache de sécuritè, destinée à maintenir la continuité mécanique du câble, au cas où il serait rompu au regard de l'isolateur, remplira son rôle en cas d'amorçage d'un arc au point de suspension.

Arrêt des conducteur's sur les isoluleurs. - La prescription d'arrèt des conducteurs sur les isolateurs formulée au deuxième alinéa du paragraphe 4 de l'article 5 est réalisée, dans les lignes établies avec isolateurs suspentus, par l'emploi de pinces de fixation retenant le conducteur sans le laisser glisser.

Parafils. - Dans le cas de lignes équipées avec isolateurs suspendus, les parafils aux appuis d'angles ne sont pas exigés; il appartient néanmoins aux exploitants de prévenir les effets de la rupture des conducteurs ou des chaînes par le renforcement de dispositifs employés couramment.

En ce qui concerne le voisinage des maisons, si on ne peut l'éviter, les croquis ci-après traduisent les dispositions à adopter pour les conducteurs au voisinage d'un toit en pente, d'un toit en terrasse et d'un toit à la mansard, pour les isolateurs rigides et pour les isolateurs suspendus.

Enfin, l'article 65 prévoit l'obligation de transpositions régulières sur les canalisations de troisième catégorie, afin d'établir une symétrie aussi grande que possible de chacun des conducteurs d'une canalisation par rapport au sol. Cette disposition a principalement pour but de réduire l'induction électriçue, qui devient parfois importante aux tensions de $3^{\circ}$ catégorie, de ces canalisations sur les lignes de télécommunication.

\section{Section II. - Sous-stalions, postes de transformation et installations diverses.}

67 \ 1 er. - Pour garantir la sécurité du personnel amené ravailler dans les locaux non gardés où sont installés des trans- formateurs de troisième catégorie, en cas d'incendie ou d'explosion, il est nécessaire que les portes, lorsqu'elles sont à rabattement, ne s'onvrent pas vers l'intérieur. Or, ces locaux sont fréquemment installés sur une voie publique ou en bordure d'une pareille voie, et les règlements de voirie interdisent généralement l'ouverture des portes vers l'extérieur. 11 est éviclent que les motifs qui ont déterminé cette disposition doivent céder devant la question primordiale de sécurité du personnel; il y aura donc lieu, en pareil cas. d'autoriser l'ouverture des portes vers l'cxtérieur en veillant toutefois à ce que la saillie qui résultera du rabattement de la porte sur le mur de façade soit réduite au minimum.

Art. 68. - A l'intérieur des sous-stations el postes de transformation, la protection résulte de distances suffisantes ménagées entre garde-corps et conducteurs, aussi bien qu'entre garde-corps et entre garde-corps et parois.

s'il est fait emploi de couches de peinture pour différencier les conducteurs, on pourra employer une couleur déterminéc pour chaque phase, quelle que soit la position respective des conducteurs, sans que cette indication doive être considérée comme imposant ce procédé de préférence à tout autre.

\section{Art. 69, \$2. -- De la prescription ainsi conçue}

"L'accès de ce passage est (léfendu par une porte f'rmant à clef", on a cru parfois pouvoir conclure que ladite porte devail rester fermée à clef en permanence. Une pareille interprétation serait erronée, car c'est avec intention (qu'il est écrit "fermant à clef " et non "fermée à clef ». La porte doit pouvoir ètre fermée à clef lorsqu'une surveillance ne peut ètre exercée sur l'accès qu'elle défend. Mais il arrivera fréquemment que les nécessités de l'exploitation obligent à la laisser ouverte, et il n'en résultera aucun inconvénient si l'accès est, pendant ce temps. l'objet d'une surveillance suffisante pour que seul le personnel qui a mission de pénétrer dans la partie arrière du tableau puisse le faire.

\section{Section V. - Dispositions spéciules applicables à la traversée des lignes de chemins de fer.}

Art. $i t .=$ Les remarques f mulées précédemmont all sujet. de l'article 39 sont égal ment appiicables à l'article 74.

\section{Sectton VI.-- Protection des lignes de télécommunication}

Art. 76 . - Le développement des canalisations de troisième catégorie, dont l'influence sur les lignes de télécommunication peut devenir importante à petite distance, oblige à prendre quelques précautions spéciales dans létablissement des projets de ces canalisations.

Pour tirer le meilleur parti des mesures à prendre pour la protection des lignes de télécommunication, et afin de faciliter leur application pratique, il est désirable que les services télégraphic|ues ou téléphoniques et les services électriques intéressés apportent la meilleure volonté de collaboration. La communication réciproque, d'une manière systématique et régulière, de tous renseignements utiles relatifs aux constructions de lignes existantes ou projetées, aux changements des conditions d'exploitation d'installations voisines est très recommandable.

Les lignes de télécommunication doivent présenter certaines conditions de bonne construction et de bon entretien, notamment en ce qui concerne la symétrie des circuits et la perditance qui doit être aussi peu différente que possible pour chacun des conducteurs du circuit et aussi petite que possible. Mais l'arrêté technique ne saurait réglementer les conditions que doivent remplir les lignes de lélécommunication : il est, au contraire, de son domaine de s'occiper des conditions que doivent remplir les canalisations d'énergie. Déjà l'article 64, paragraphe 5, prévoit l'obligation de transpositions régulières sur les canalisations de troisieme catégorie. L'article 76 va plus loin et précise que toul. projet doit être accompagné d'une évaluation des lensions incluites sur les lignes de télécommunication avoisinantes, en même temps qu'il précise certaines conditions relatives aux transpositions.

Les méthodes de détermination des tensions induites ne sont précisées ni dans l'arrêté, ni lans la présente circulaire. Elles feront l'objet, s'il y a lieu, d'instructions ultérieures.

Ainsi que l'indique le titre de l'article 76, les dispositions de cet article ne sont pas applicables aux lignes existantes. 
Art. 7\%. - L'indication d'une distance minimum entre les canalisations d'énergic et les lignes de télécommunication n'exclut nullement l'adoption d'un plus grand écartement s'il est pratiquement et raisomnablement réalisable.

Le premier alinéa du paragraphe 2 spécifie qu'aux points de eroisement les conducteurs d'énergic sont toujours placés au-dessus des fils de télécommunication.

Croisements abe les lignes de téléconmunicalion. -... Lorsqu’il ne sera pas substitué de fils souterrains aux fils de télécommunication, des câbles de garde seront installés au-dessus des circuits de télécommunication.

Ce dispositif de protection pourra consister en un ou plusieurs câbles d'acier galvanisés d'une section suffisante qui, en aucun cas, ne pourra être inférieure à $30 \mathrm{~mm}^{2}$, mis au sol, soutenus par des supports métalliques ou en bois à une hauteur suffisante et parallèlement aux fils de télécommunication.

Art. 78 . - Une exception aux prescriptions de l'article 78 a été apportée en ce qui concerne les parties de lignes de télécommunication montées sur des supports particuliers et suffisamment séparées électriquement des parties montées sur les mêmes appuis que la canalisation d'énergie.

\section{Section VII. - Entrctien des onnrages. - Exploilation des distributions.}

Art. 79. - Les remarques formulées à propos de l'article 60 s'appliquent également à l'article 79 .

\section{TITRe II. - INSTAllations de thaction Électrieve.}

les dispositions applicables aux installations de traction électrique onl été réunies dans le tilre II, qui comporte les divisions suivantes :

Chapitre $I^{\text {er }}$ - - - Disposilions applicables à la fois à la traction par courant continu el par courant alternatif.

Chapitre II. - - Dispositions spéciales à la traclion par courant continu.

Chapitre 111 . - Dispositions spéciales à la traction par courant alternatif.

Il est à peine besoin de signaler que la stricte observation de ccs prescriptions ne suffit pas à exonérer les entrepreneurs de leurs responsabilités vis-à-vis des tiers auxquels leurs installations viendraient à causer des dommages. soit par induction, soit par électrolyse, soit pour tout autre motif.

Ces prescriptions, en effet, dont le but est d'éviter, dans la plupart des cas, que l'entreprise de traction ne cause des donmages aux tiers, ne sauraient avoir pour résultat d'éviter ces dommages dans tous les cas. Dans les cas particuliers où de pareils dommages se produiraient, il y aura lieu, pour l'entreprise de traction, d'indemmiser les tiers auxquels dommage aurait été causé.

\section{Cil. ier. - Traction par colmants de toute espece (continu et alternatif)}

\section{Section I. - Lignes de contact}

Drviston I. - Dispositions communes.

Art. 82 . - En raison des conditions de leur installation et de leur exploitation, les ouvrages des entreprises de traction bénéficient des tolérances admises pour l'établissement des installations de première catégorie tant que la tension entre les conducteurs et la terre ne dépasse pas $1.500 \mathrm{v}$. cn cas de courant continu et $600 \mathrm{v}$. en cas de courant alternatif. L'établissement des ouvrages servant à la traction par l'électricité est ainsi facilité autant que le permet le souci de la sécurité.

L'entretien des ouvrages pour ]esquels cetle tolérance est admise devra être assuré avec un soin tout particulier.
"'Arl. 83. - Il est bien entendu que chacun des isolateurs mis en série ou chacune des cloches doit remplir les conditions d'isolement requises pour la tension considérée.

\section{Division II. - Installation de traction empruntant la voie publique.}

Art. 85. - La prescription relative à la hauteur de $6 \mathrm{~m}$. est parfois difficile à respecter. Les passages inférieurs sous lesquels doit passer la ligne ont en effet fréquemment des hauteurs beaucoup moindres, de sorte que l'appareil de prise de courant doit fonctionner convenablement à des hauteurs très différentes; l'écart devient si grand entre les positions extrêmes qu'il amène parfois à des constructions presque irréalisables. Il n'a pas sєmblé, toutefois, qu'il y eût lieu de prévoir la réduction de la hauteur de $6 \mathrm{~m}$. par une disposition générale, mais des dérogations pourront ètre demandées chaque fois que la hauteur habituelle de chargement des véhicules permettra d'envisager un abaissement de la hauteur régiementaire, et il est bien entendu que la demande de dérogation pourra s'appliquer à l'ensemble d'un réseau.

Le paragraphe $1^{\text {er }}$ autorise les traversées des voies publiques par des fils de contact de deuxième catégorie à une hauteur comprise entre 6 et $8 \mathrm{~m}$. pourvu que la traversée comporte, dans ce cas, un dispositif apparent d'avertissement.

Il n'a pas paru nécessaire, ni avantageux pour l'industrie, de préciser dès à présent quelles formes pourra revêtir ce dispositif d'avertissement, qui ne sera pas, à proprement parler, un dispositif de protection, mais devra signaler clairement la traversée afin qu'elle ne soit pas apercue trop tardivement par les usagers de la voie publique. Il appartiendra au service du contrôle de concilier, dans l'examen des propositions qui seront présentées, la nécessité d'avertissement ainsi reconnue avec le devoir de ne pas imposer à l'entreprise des obligations qui ne soient pas pleinement justifiées

Il est signalé à propos du paragraphe 2 qu'il est in utile d'€xice le remplacement des fils de contact de section inférieure à $30 \mathrm{~mm}$ qui pourraient exceptionnellement être en service dans les instal lations existantes, si le faible diamètre de ces fils n'entraîne par d'accidents dans ces installations.

Art. 86. - L'article 86 admet une réduction des coefficients de sécurité des lignes de contact de deuxième catégorie. Le coefficient 3 prévu pour les lignes d'alimentation entraîne, en effet, des flèchcs incompatibles avec le bon fonctionnement de l'appareil de prise de courant. Il va sans dire que l'entretien des lignes qui jouiront de cette dérogation devra être assuré avec un soin pasticulier.

\section{Drviston III. - Voies établies sur plate-forme indérendante.}

En principe, les installations auxquelles s'applique le texte sont supposées établies sur traverses en bois et hallast, en admettant bien qu'il ait paru inutile de maintenir cette presciption dans le corps même de l'arrèté, que le ballast est, autant que possible, disposé de manière à ne pas toucher les rails et à ne pas recouvrir les traverses en bois.

Ce cas comprend la presque totalité des voies ferrées d'intérèt général, les lignes de chemins de fer métropolitains, tels que le chemin de fer métropolitains et le chemin de fer souterrain NordSud de Paris, la plupart des voies ferrées d'intérèt local, et peut s'appliquer également, mais à titrc exceptiomnel, à certains tramways suburbains ou interurbains.

Les principales différences avec les entreprises empruntanit la voie publique résultent, d'une part de ce que les rails de runlement peuvent ctre beaucoup mieux isolés de la terre, d'autre part, de ce que ces rails sont en général beaucoup plus éloignés des conduites métalliques susceptibles de souffir de phénomènes électrolytiques et, enfin, de ce que les courants employés sont, dans la plupart des cas, beaucoup plus intenses.

Les deux premières conditions permettent d'admettre des chutes de tension dans les rails beaucoup plus élevées que dans les installations empruntant la voie publique, ce que rend d'ailleurs indispensable la troisième condition. Il n'a même pas paru possible de limiter ici ces chutes de tension par des nombres précis, mais seulement par la condition qu'il n'en résulte aucun inconvénient. 
Dans les installations empruntant les voies publiques, et sous réserve des cas très exceptionnels où la prise de courant se fait en caniveau souterra!n, les lignes de contact sont forcément aériennes.

Dans les cas d'emploi d'une plate-forme indépendante, la ligne de contact pent ètre soit aérienne, soit à fleur du sol ; on a, dans ce dernier cas, donné au conducteur de contact la dénomination spéciale du "rail de contact" (art. 97) en réservant le nom de "fil de contact" (art. 87 ) au cas de la ligne aériemne.

Il est bien entendu que, pour les installations de roies ferrées ćtablies, sur presque toute leur longueur, sur plate-forme indépendante et empruntant ou ne eroisant la voie publique qu'en certains points relativement distants, comme c'est la cas, par exemple, des passages à niveau sur les voies ferrées d'intérêt général, les prescriptions édictées par le chapitre $I^{\text {er }}$ du titre II concernant les installations de traction sur voie publique ne seront applicables qu'aux points d'emprunt ou de croisement.

Art. $8 \%$. - Les hauteurs minima de $5 \mathrm{~m} .50$ et de $6 \mathrm{ml}$. ont été maintenues. Toutefois, dans les départements où ces hauteurs pourraient ètre réduites sans inconvénient, il y aura lieu d'établir des demandes de dérogation dans les conditions qui viemnent d'être indiquées à propos de l'article 85 .

\section{Section II. - Ulilisation des rails de roulement comme conducteurs de courant.}

Art. 89. - Il importe que le service du contrôle assure strictement l'exécution de toutes les mesures jugées nécessaires dans chaque cas pour protéger contre l'action nuisible des courants dérivés les masses métalliques voisines de la ligne de traction et notamment les lignes de télécommunication ainsi que les autres lignes électriques.

Les lignes de télécommunication peuvent être unifilaires; les dispositions de l'article 89 s'appliquent en principe à ces lignes. Il peut cependant arriver qu'il y ait de réelles difficultés à protéger ces lignes unifilaires, alors que la protection de circuits bifilaires n'empruntant ni le sol ni les voies serait aisée et que l'établissement de pareils circuits bifilaires ne présente aucune difficulté sérieuse. En pareil cas, c'est à l'auteur des troubles produits dans ces circuits à les faire disparaître, mais s'il est reconnu que le procédé efficace le plus économique soit d'adopter un double fil, le possesseur des circuits unifilaires ne pourra pas s'opposer à l'application de ce procédé.

Arl. 92 et 95 . - Les articles 92 et 95 précisent certaines dispositions nécessaires à la surveillance des réseaux. Dans le cas où les joints des rails de roulement sont soudés, la vérification de la conductance des joints pourra être remplacée par la mesuri de la conductance de longueurs de voies comprenant chacune au moins un joint.

Art. 93. - L'article 93 divise les réseaux en une zone urbaine et une zone suburbaine. Cette division, qui répond à la réalité dans la plupart des entreprises de traction se justifie aisément par la double considération que les prescriptions imposées dans la zone urbaine deviennent facilement prohibitives si on les applique à de longues prolongations suburbaines, alors qu'elles se justifient moins parce que les dangers d'électrolyse, notamment, deviennent moindres, dans la plupart des cas, pour ces lignes suburbaines.

La répartition des lignes entre la zone urbaine et la zone suburbaine devra donc être faite avec le plus grand soin, lors de l'instruction des projets. Cette répartition sera toujours revisable d'un commun accord entre le concessionnaire et le service du contrôle ; en cas de désaccord, le dossier me sera renvoyé et je statuerai, après avis du Comité d'Electricité. Je signale à ce sujet qu'en vue d'avantager les lignes suburbaines, pour lesquelles les dangers d'électrolyse sont notablement diminués, les chutes moyennes de tension seront déterminées, non plus pendant la durée effective de la marche normale des voitures, mais sur une période de vingtquatre heures consécutives. Cette manière de procéder supprimera, en outre, les contestations qui s'élevaient très souvent à propos de l'exploitation des lignes suburbaines, dont le trafic est généralement peu important. Pour les lignes urbaines, les chutes moyennes de tension continueront à ètre calculées sur la durée effective du service.
Cit. 11. - Dispositions spécrales a la thaction par courant CONTINU.

\section{Section II. - Utilisation des rails de roulement comme conducteurs de courant.}

Division 1. - Installations de traction empruntant la voic publicue.

Art. 98. - - Je signalerai tout d'abord, en attirant l'attention sur les indications du paragraphe $I^{e r}$, la nécessité, pour le service du controle, d'exiger de la part des entreprises qui utilisent les rails comme conducteurs de courant, la vérification périodique de la conductance de la voie, qui peut être faite tout d'abord par grandes longueurs, puis par sections plus petites, si le résultat n'est pas satisfaisant, jusqu’à ce que l'on ait trouvé les points où l'ćclissage électrique est défectueux.

Art. 10\%.-.- Le paragraphe 2 précise la question des comnexions transversales. Il est bien entendu que des voies placées sur accotement de part et d'autre d'une voie publique ne seront pas considérées comme juxtaposées.

Art. 10\%. - L'article 10.1 indique les chutes de tension mojennes par kilomètre et précise, en mème temps, que cette perte de charge doit ètre effectivement mesurée sur un kilomètre de voie et non pas sur une longueur arbitraire dont la chute de tension serait ensuite ramenée à la perte de charge kilomètrique par une règle de trois. Ces mesures donnent, sur des longucurs quelconques, des résultats qui peurent ètre très différents de la perte de charge sur un kilomètre et mème très différents les uns des autres selon la longucur de voie adoptée pour l'essai sur un même kilomètre.

l.e paragraphe 2 de larticle 10.1 permet cles clérogations lorsque les conchuites métalliques s'éloignent des rails, sous réserve qu’il n'en résulte aucun inconvénient. Ces dispositions découlent naturellement du fait que la densité du courant de retour dans le sol décroît rapidement à mesure que l'on s'écarte du rail ; clles résultent d'essais expérimentaux el ne paraissent pas devoir présenter d'inconvénients si les réserves formulées sont observées exactement.

Art. 105. - L'article 105 précise le degré d'équipotentialité qu'il y a lieu d'exiger entre les points de comnexion des arteres de retour avec les rails. Il ne parait, en effet, ni nécessaire, ni mème toujours avantageux, de réaliser une équipotentialité parfaite de ces points de connexion et il est préférable de fixer une limite à leur différence de potentiel moyenne.

Drvision II. - Voies établies sur plate-forme indépendante.

Lorsque des installations de traction établies nomalement sur plate-forme indépenclante emprunteront exceptionnellement la voie publique soit sur des longueurs relativement faibles, soit en des points distants les uns des autres, comme c'est le cas des passages à niveau, les prescriptions de la division. lls pourront, en princlpe, rester applicables aux installations faites sur la voie publique ; mais dans les cas où ces emprunts présenteraient une importance particulière soit par suite de leur longueur, soit par suite de la présence de canalisations ou masses métalliques souterraines, il appartiendrait an service du contrôle d'examiner dans chaque cas d'espèces, avec les autres services intéressés et avec le concessionnaire, les dispositions que la sécurité pourrait exiger.

Art. 106.-- Du texte de cet article, il résulte que les dispositions du paragraphe 2 de l'article 98 et de l'article 94 ne sont pas applicables aux voies établies sur plate-forme indépendante. Il est. nécessaire, en effet, de laisser subsister, en ce qui concerme ces voies, une souplesse suffisante dans les prescription relatives aux connexions transversales, en raison de l'emploi possible des rails de roulement pour la signalisation, et dans les prescriptions relatives à l'issolement par rapport aux ouvrages métalliques, en raison des cas très variés qui peuvent se présenter.

Cir. III. - Dispositions spéciales a ta traction palk Couran't ALTERNATIF.

I.e chapitre III précise les prescriptions applicables aux inslallations de traction électrique par courant altematif, qu'elles soient établies sur voie publique ou sur plates-formes indépendantes. 
Les installations de traction clectricue par courant alternatil établies sur voies publicues ou sur plate-forme indépendante ne présentent pas de dilférences importantes; les prescriptions qui les concernent ont été réunies en un seul chapitre.

Les dispositions relatives aux lignes aériennes de contact (section 1) restent à peu près les mêmes que celles se rapportant aux autres systènes de traction.

L'article 3 prévoit des mesures spéciales pour supprmer le courant sur certaines sections de voie non parcourues de façon permanente par les trains, lorsque les mancuvres n'y sont pas nécessaires.

Les prescriptions de la section II sont sensiblement différentes de celles prévues pour l'utilisation des rails de roulement comme conducteurs de courant dans le cas des installations à courant continu.

Un certain nombre de mesures a été préconisé pour maintenir la conductance des rails (art. 112 et 113).

Des dispositions spéciales devront, en cas de besoin, être réalisées pour éviter les perturbations nuisibles au bon fonctionnement des lignes de télécommunication situées au voisinage de la voie.

Il est, en effet, incontesté que, d'apres la législation existante, les circuits électriques de traction ne doivent pas créer de perturbation nuisible sur ces lignes; en particulier, l'article 17 de la loi du 15 juin 1906 donne à l'administration des Postes et des Télégraphes, dans son premier alinéa, et aux fonctionnaires chargés de la surveillance de tout service public, dans son deuxième alinéa, le droit de réquisition à l'effet de prendre toutes mesures nécessaires pour prévenir ou faire cesser de pareilles perturbations.

Il est donc de lintérêt commun du concessionnaire et des services publics intéressés que l'étude des perturbations possibles soit faite avant la mise en exploitation des installations et que les mesures nécessaires aient été prises pour éviter dans la mesure où les prévisions sont possibles, toute perturbation nuisible sur les lignes voisines.

Divers procédés ont été préconisés dans ce but, mais leur efficacité n'a pas été assez nettement établie à ce jour pour qu'ils puissent faire l'objet de dispositions réglementaires. Il y aura lieu, en conséquence, de faire l'étude de chaque installation de ce genre à titre de cas d'espèce.

Art, 114, - L'obligation d'installer des plaques de terre sur les rails a été supprimée, les rails se trouvant suffisamment reliés au sol par construction.

La section III précise quelques prescriptions de sécurité applicables au matériel roulant.

\section{Titre III. - Dispositions Diverses.}

Le titre III contient diverses dispositions nécessaires pour l'application de l'arrêté. Les dispositions de l'arrèté ne sont pas limitatives. Lorsque les circonstances locales l'exigent, le service du contrôle peut imposer, pour l'établissement des installations, toutes les mesures nécessaires pour assurer la sécurité.

Dans cet ordre d'idées, je citerai, par exemple, les conditions d'implantation et d'établissement des lignes, notamment en pays de montagne, au point de vue des mesures à prendre contre les dangers que peuvent présenter éventuellement les éboulements, les torrents, les avalanches, etc.

J'indiquerai également le voisinage de certains établissements dangereux, tels que les fabriques ou dépôts d'essence, benzine, éther, acétylène, amorces fulminantes, etc., dont la proximité peut exiger des précautions spéciales. Il n'a pas paru possible, à cause de leur diversité et des différences qu'ils comportent, d'assimiler ces établissements aux poudreries et magasins à poudre visés à l'article 121, mais il doit ètre bien entendu que l'on s'efforcera d'en éloigner toute cause de danger et que chaque cas fera l'objet d'une étude particulièrement attentive.

On remarquera, d'autre part, que l'article 121 ne vise pas mon plus les chambres ou dispositifs de mine établis dans certains ouvrages d'art, mais qui ne sont pas chargés en temps de paix. Tant qu'elles demeurent vides, en effet, ces chambres ne constituent pas des magasins à poudre proprement dits et ne doivent donner lieu à aucune mesure spéciale quant au roisinage des lignes d'énergie. Toutefois, on devra signaler aux services compétents l'établissement de ces dernières, lorsqu'elles s'en approcheront plus près qu'il est indiqué dans ledit article.

Art. 122. - J'attire enfin votre attention sur les conditions d'application fixées par cet article.

Comme il peut $\mathrm{y}$ avoir avantage, en certains cas, à équiper tout ou partie d'une installation relevant d'une catégorie suivant les règles correspondant à une catégorie supérieure (par exemple pour bénéficier du deuxième alinéa du paragraphe 1 er de l'article $\overline{7} 4$, relatif à la traversée des chemins de fer), l'entrepreneur de l'installation pourra le faire, mais à la condition d'observer pour les parties ainsi traitées toutes les règles applicables à cette catégorie supérieure.

Le paragraphe 2 du mème article 122 maintient la possibilité de dérogations accordées par le ministre dans certains cas motivés, après avis du Comité d'Electricité. Mais, comme ces dérogations peuvent soulever des objections de la part de divers services intéressés, il a paru nécessaire. pour le cas où ceux-ci n'auraient pas donné leur adhésion, de prévoir qu'avant de statuer, le ministre pourra s'éclairer des autres avis qu'il jugerait utiles.

Le paragraphe 3 n'a pas été modifié ; il permet aux services de contrôle d'imposer, lorsque la sécurité l'exige, des conditions spéciales justifiées par les circonstances. Mais cette faculté demeure exceptionnelle, elle ne doit pas ètre considérée comme avant pour but de permettre l'addition au texte du présent arrêté de prescriptions supplémentaires d'ordre général. dont l'adoption ne saurait intervenir qu'après avis du Comité d'Electricité.

\section{Le ministre des Travaux publics,}

André Tardiet. 